This item was submitted to Loughborough's Research Repository by the author.

Items in Figshare are protected by copyright, with all rights reserved, unless otherwise indicated.

\title{
Experimental study of multiple-channel automotive underbody diffusers
}

PLEASE CITE THE PUBLISHED VERSION

PUBLISHER

Professional Engineering Publishing / @ IMECHE

VERSION

VoR (Version of Record)

LICENCE

CC BY-NC-ND 4.0

REPOSITORY RECORD

Jowsey, Lydia, and Martin A. Passmore. 2010. "Experimental Study of Multiple-channel Automotive Underbody Diffusers". figshare. https://hdl.handle.net/2134/6523. 
This item was submitted to Loughborough's Institutional Repository (https://dspace.lboro.ac.uk/) by the author and is made available under the following Creative Commons Licence conditions.

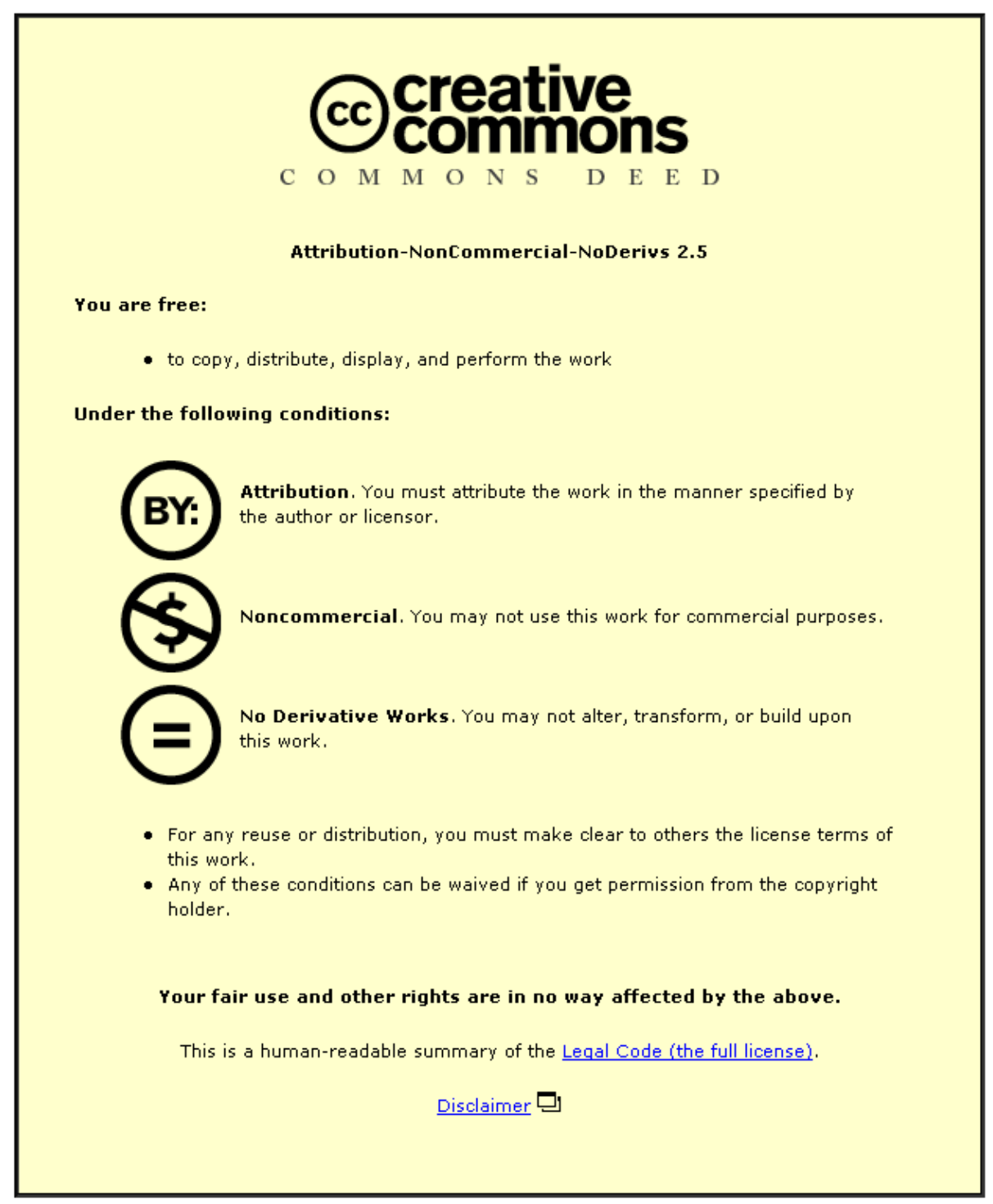

For the full text of this licence, please go to: http://creativecommons.org/licenses/by-nc-nd/2.5/ 


\title{
Experimental study of multiple-channel automotive underbody diffusers
}

\author{
L Jowsey* and M Passmore* \\ Department of Aeronautical and Automotive Engineering, Loughborough University, Loughborough, Leicestershire, UK
}

The manuscript was received on 6 August 2009 and was accepted after revision for publication on 16 March 2010.

DOI: 10.1243/09544070JAUTO1339

\begin{abstract}
Underbody diffusers are used widely in race car applications because they can significantly improve the cornering capacity of the vehicle through the generation of a downforce. They are also likely to have a wider role in reducing the drag in road vehicles as it becomes increasingly important to reduce emissions of carbon dioxide.

This paper reports on a wind tunnel investigation, using a simplified bluff body model, into the effect of splitting a simple plane diffuser into multiple channels. Tests are reported for a range of diffuser geometries suitable for road and race car applications. The results for the lift, the drag, and the incremental changes to the lift-to-drag ratio are reported and discussed in terms of the underbody pressures.

While broadly similar trends to the single-channel plane diffuser are seen in the multiplechannel diffuser configurations, it was found that the effect of increasing the number of channels depended on the flow regimes present in the plane diffuser. At angles just above the plane diffuser optimum, where the flow is partially separated, the multiple-channel configurations give large improvements in the downforce with minimal increase in the drag, significantly extending the performance envelope. The pressure maps indicate that the gains occur through improved diffuser pumping and pressure recovery in both the inner and the outer channels.
\end{abstract}

Keywords: multiple-channel automotive underbody diffusers, wind tunnel, simplified bluff body model

\section{INTRODUCTION}

Aerodynamics and airflow management are important in both passenger and race car design, as an aerodynamically optimized car can give a better performance through improved handling, stability, and fuel consumption. In road vehicle applications the emphasis since the 1970s has been the need to reduce the drag of vehicles [1]. This is again high profile with the drive to reduce carbon dioxide. In race car design the emphasis is on a reduction in the lap times and, while reducing the drag can have some benefit, the lift and the lift distribution have much greater influences. An increase in the downforce

*Corresponding author: Department of Aeronautical and Automotive Engineering, Loughborough University, Stewart Miller Building, West Park, Loughborough, Leicestershire LE11 3TU, UK.

email: L.Jowsey@lboro.ac.uk; m.a.passmore@lboro.ac.uk (negative lift) can result in improved acceleration times and reduced braking distances, because the increased normal load can improve traction, but more importantly it allows the generation of a larger lateral force and hence higher cornering speeds. In addition, the distribution of the downforce influences the cornering ability, because the relationship between the centre of pressure and the centre of gravity determines the oversteer-understeer characteristic. While the benefits of an increased downforce are clear, an increase in the downforce generally results in a corresponding increase in the drag and it is therefore essential to produce an appropriate compromise.

The design of the underbody diffuser, where one is employed, can have a significant influence on the drag, the downforce, and the downforce distribution, with the diffuser on a Formula 1 car contributing up to 40 per cent of the overall downforce [2].

Published work on automotive diffusers has generally concentrated on detailed studies of simple 
plane diffusers. The most comprehensive set of results were reported by Cooper et al. [3, 4] on a diffuser-equipped bluff body; they identified three downforce mechanisms: upsweep, ground interaction, and diffuser pumping. Ground interaction occurs because, as a body is brought to the ground, the underbody flow is constrained, causing flow acceleration and a decrease in the static pressure, which suppresses the pressure recovery after the lower front radius and increases the downforce. This increase continues until a point at a very small ride height where the effect of the fluid viscosity becomes dominant and no further increase in the downforce is achieved. Diffuser pumping occurs because the exit pressure of an automotive diffuser is essentially fixed by the vehicle base pressure. As the pressure is recovered along the length, the fixed exit pressure effectively reduces the diffuser inlet pressure. This depression is a significant source of the downforce but also has the effect of reducing the flat underbody pressure. The reduction in the pressure on the flat underbody has been found to produce a larger proportion of downforce than the diffuser does [4], but it is the diffuser that controls how much the pressures are 'pumped down'. The underbody upsweep aids downforce production in a similar way to that of an inverted wing, and a diffuser without end plates can be compared with an inverted fastback. In a fastback the presence of trailing vortices produces a downwash which helps to maintain attached flow [5] whereas in a diffuser these vortices create an upwash. The results of Cooper et al. showed a general trend of increasing the downforce with increasing angle and decreasing ride height, to a maximum. An analysis of centre-line pressure data showed that the flat underbody upstream of the diffuser dominates the downforce generation, with the pressure on the flat floor driven by the ground effect and diffuser pumping.

Howell [6] investigated a simple wheel-less model with interchangeable backlight and diffuser angles and found similar trends to those obtained by Cooper et al. The maximum downforce was generated in the square back configurations $\left(0^{\circ}\right.$ and $\left.40^{\circ}\right)$ with the downforce reducing as the back angle increased from $0^{\circ}$ to $30^{\circ}$. Additionally, the optimum diffuser angle for drag was found to be highly dependent on the overbody shape.

George and Donis [7] investigated a plenum and venturi diffuser configuration using sealed skirts (touching the ground) and open skirts (not touching the ground). Small angles $\left(5^{\circ}\right)$ were seen to perform well with sealed skirts whereas larger angles $\left(10^{\circ}\right.$ and $15^{\circ}$ ) worked better with open skirts. They also identified a pair of longitudinal vortices present in the diffuser channel that became stronger with increasing diffuser angle. At very high ride heights these vortices disappeared.

Zhang and co-workers [8-10] undertook several investigations using a diffuser model similar to that employed by Cooper et al. with a $17^{\circ}$ diffuser angle $[3,4]$ and a range of angles between $5^{\circ}$ and $20^{\circ}[10]$. Force, pressure, laser Doppler anemometry, and surface flow visualization measurements were reported. Similar trends in downforce production and pressure distributions to those reported by Cooper $e t$ al. [3] were observed. The surface flow visualizations provided good insight into the flow mechanisms with the identification of streamwise vortices, separation bubbles, as well as the change in these mechanisms with diffuser angle and ride height, showing vortex breakdown and asymmetry.

A broad interpretation of the published literature is that, as the diffuser area ratio (AR) is increased, the depression associated with diffuser pumping deepens, driven by the vortices emanating from the diffuser inlet. The vortex strength increases with increasing AR, aiding flow attachment, which ensures diffuser operation up to relatively large angles compared with two dimensions. At more extreme ARs the increasingly adverse pressure gradient forces partial or complete flow separation and vortex breakdown, causing a reduction in the downforce.

This paper aims to investigate the performance of multiple-channel diffusers and to make a comparison with a single-channel plane diffuser. The use of multiple channels is seen on many road and race applications either as a result of packaging restrictions or in response to regulations, but there is a lack of published work available on their performance when compared with single-channel plane diffusers. The work uses a model based on that of Cooper et al. [3] fitted with a plane diffuser that can be subdivided into two, three, or four channels. A wider range of diffuser angles (from $0^{\circ}$ to $30^{\circ}$ ) than those reported to date in the literature are investigated to cover more of the range practically encountered in race applications. The range of ride heights investigated covers a range of on-road applications between $16 \mathrm{~mm}$ and $44 \mathrm{~mm}$ corresponding to a non-dimensional ride height $h_{1} / H$ range of $0.0516-0.1419$, where $h_{1}$ is the ride height and $H$ is the model height.

\section{EXPERIMENTAL METHOD}

The testing was performed in the Loughborough University $1.9 \mathrm{~m} \times 1.3 \mathrm{~m}$ open-circuit closed-work- 
ing-section wind tunnel. All tests were conducted at a wind speed of $40 \mathrm{~m} / \mathrm{s}$, and the force and moment measurements were taken using a six-component underfloor balance. The pressure measurements employed a 64-channel high-speed pressure scanner. The repeatability of the measurements following a complete dismantle, removal, and reinstallation of the model is \pm 0.009 for the drag coefficient $C_{\mathrm{D}}$ and \pm 0.035 for the lift coefficient $C_{\mathrm{L}}$. However, the repeatability of the difference between two diffuser configurations after this reinstallation is \pm 0.003 for $C_{\mathrm{D}}$ and \pm 0.020 for $C_{\mathrm{L}}$. All coefficients are non-dimensionalized using the frontal area $A$.

The Loughborough University wind tunnel is not equipped with a moving ground plane and so it is apposite to consider the effects of investigating the performance of a vehicle equipped with an underbody diffuser at relatively small ground clearances using a fixed floor. George and Donis [7], Howell [6], and Cooper et al. [4] all performed tests with both fixed and moving floors and concluded that a fixedground simulation is perfectly adequate for investigating trends and mechanisms but that, if the magnitudes are required, a moving ground is essential. Cooper et al. [3] went further by plotting the optimum diffuser downforce and optimum overall downforce loci for both a fixed-ground simulation and a moving-ground simulation. These showed that, while the fixed ground under-predicted the downforce by around 10 per cent, the trends were similar, and that the optimum ARs were almost identical for the two ground simulations. Additionally, Cogotti [11] stated that, if the full moving belt and rotating wheels were not available, then the second-best simulation was a fixed ground. It is therefore concluded that in this comparative study the fixed floor is sufficient.

The model depicted in Fig. 1 is a generic bluff body equipped with a 25 per cent diffuser. The overall dimensions are a length of $800 \mathrm{~mm}$, a width of $400 \mathrm{~mm}$, and a height of $310 \mathrm{~mm}$, giving a blockage ratio in the $2.5 \mathrm{~m}^{2}$ working section of 5 per cent. The model dimensions were chosen to have a similar length:width:height ratio to that used by Cooper et al., allowing an ease of comparison; the model employed by Cooper et al. had an overall length of $396 \mathrm{~mm}$. Additionally, the use of a bluff body with a substantial base area ensures reasonable independence between the underbody and overbody flows as was shown in reference [6], where changing the slant angle (and therefore the base area) changed the optimum diffuser angle.

The diffuser plate is hinged using the arrangement shown in Fig. 2(a) with the connection to the main model made $40 \mathrm{~mm}$ upstream of the smooth hinge. This arrangement ensures that there is no backwardfacing step at the inlet to the diffuser which might trigger separation. The hinge itself is covered with a
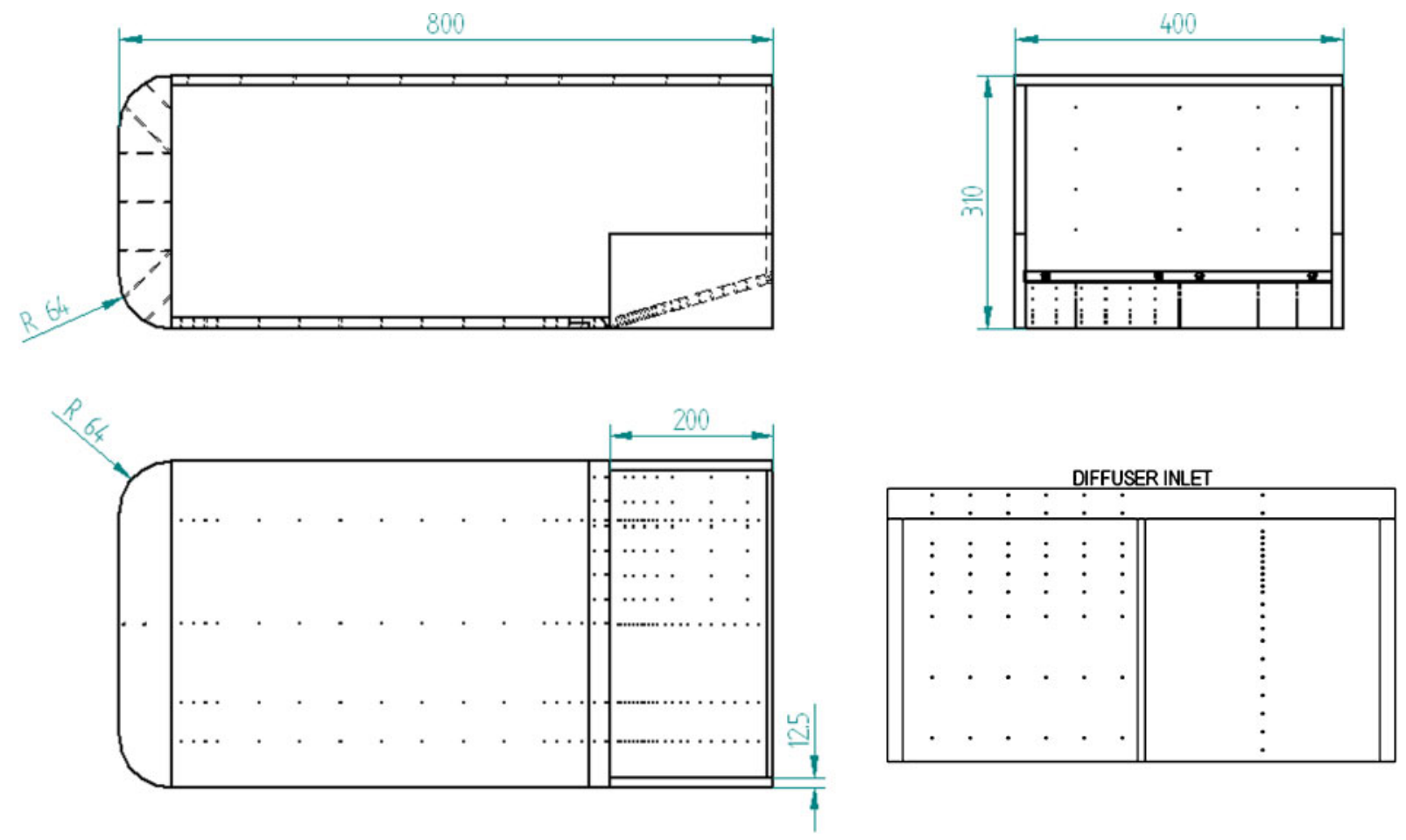

Fig. 1 Model schematic diagrams of the plane configuration 


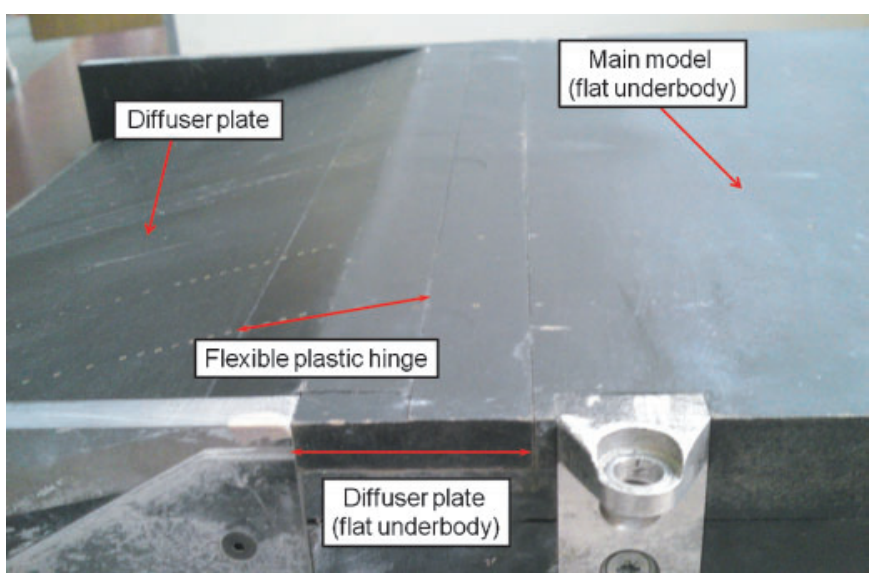

(a)

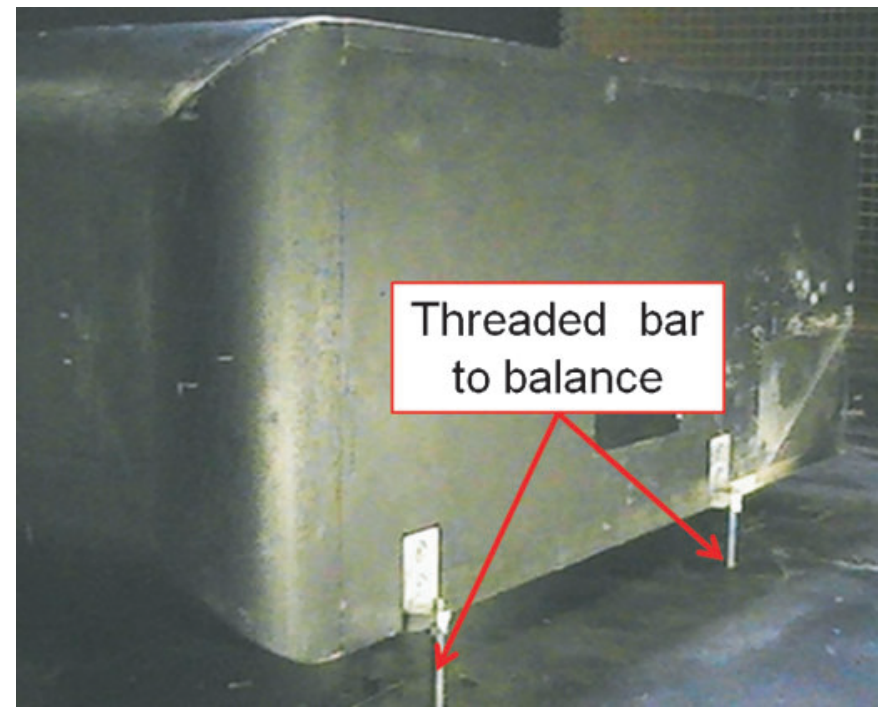

(b)

Fig. 2 (a) Hinge arrangement; (b) model attachment

thin flexible plastic skin. A positive location is provided on the model for the diffuser plate to ensure consistent diffuser angles. In the multiple-channel configurations, splitter plates are fixed within the model and the diffuser plate itself is split into the corresponding number of channels. The model is attached to the underfloor balance via a threaded bar, as depicted in Fig. 2(b).

To satisfy the need for pressure measurements, pressure tappings were located along the model centre-line (the plane channel centre-line) and along the centre-line of each diffuser channel such that rows of tappings were placed on the centre-line of the two-channel, three-channel, and four-channel diffusers. This allowed measurements to be made on the centre-line of each channel as well as a comparison of the pressure at the corresponding lateral locations when in the plane configuration. In addition, the diffusers were pressure tapped to allow area pressure maps to be produced. The plane and two-channel diffusers had one half of the diffuser tapped, while the three- and four-channel diffusers both had an outside and an inside channel tapped. An example (for the two-channel diffuser) is shown in Fig. 1.

Results are presented in the form of non-dimensional coefficients as functions of the diffuser angle $\alpha$, the non-dimensional ride height $h_{1} / H$, the nondimensional length $N / h_{1}$, and the diffuser AR given by

$$
\mathrm{AR}=1+\frac{N}{h_{1}} \tan \alpha
$$

These are shown in Fig. 3. The use of the AR is advantageous because it takes into account all variables associated with a diffuser such as the ride height $h_{1}$, the diffuser length $N$, and the diffuser angle $\alpha$. The AR is defined as the ratio of the area at the exit to the area at the inlet, which translates, in the plane diffuser case, to a ratio of heights according to

$$
\mathrm{AR}=\frac{A_{2}}{A_{1}}=\frac{h_{2}}{h_{1}}
$$

\section{PLANE DIFFUSER PERFORMANCE}

Results for the simple plane diffuser are presented in Fig. 4. For a fixed diffuser angle, the general trend of increasing downforce with decreasing ride height followed by a sharp decrease is consistent with

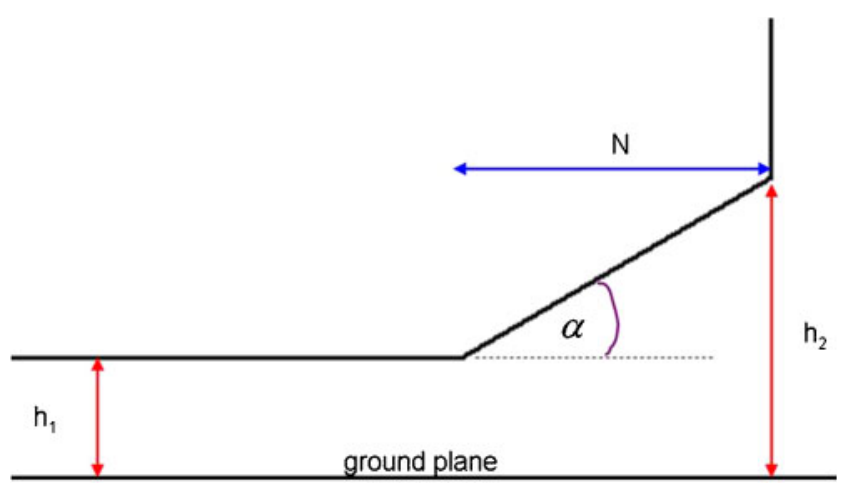

Fig. 3 Diffuser parameters 


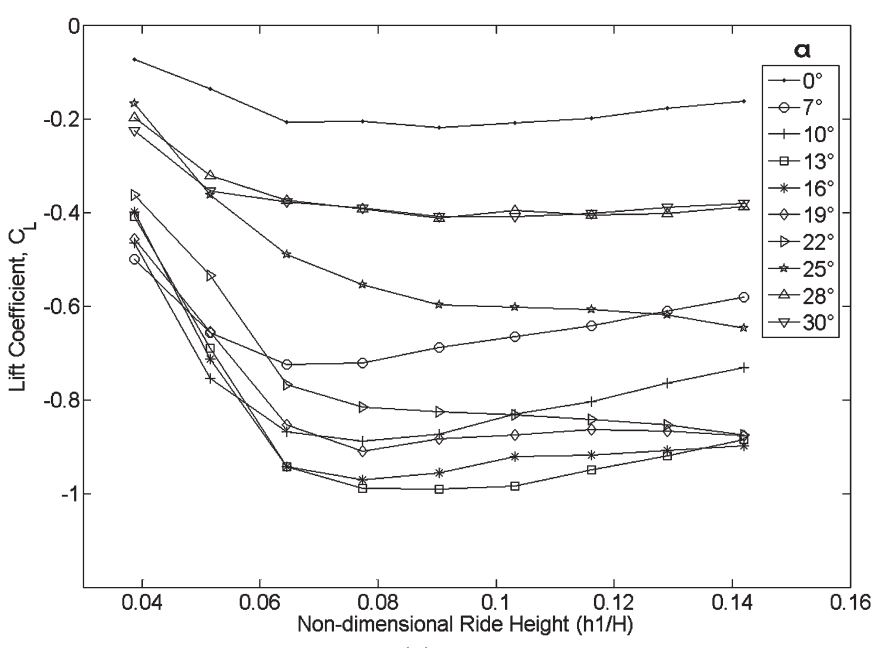

(a)

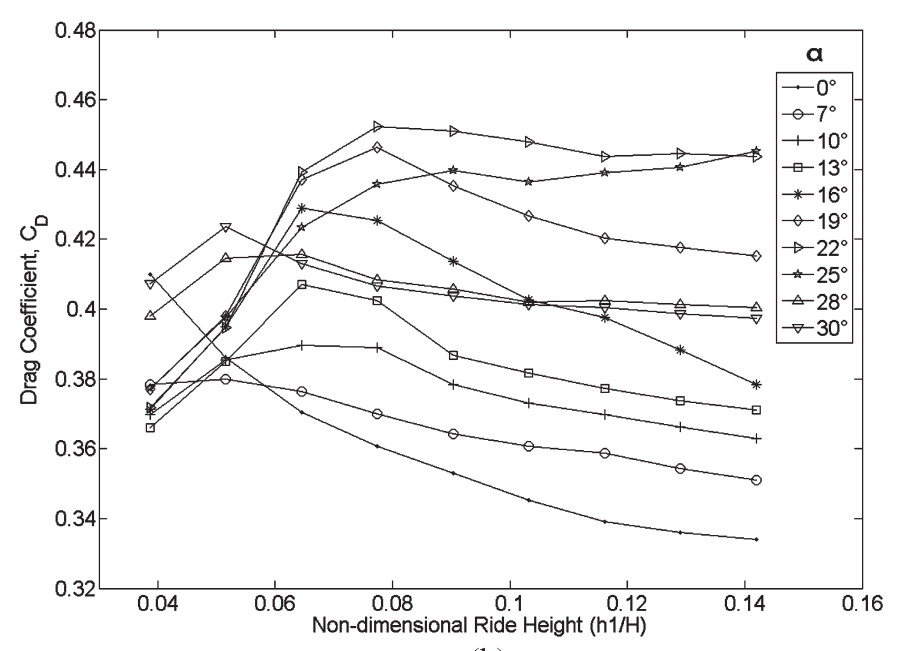

(b)

Fig. 4 Graphs of (a) the lift and (b) the drag coefficient against the non-dimensional ride height for the plane diffuser

results reported in references [3] and [8]. This sharp reduction in the downforce is accompanied by a reduction in the drag, suggesting that it arises as the viscous effects restrict the underbody flow rather than as a result of diffuser separation.

The variation between angles at particular ride heights is also significant. As the diffuser angle is increased from the flat floor $\left(0^{\circ}\right)$, an increase in the downforce is observed up to a maximum at $13^{\circ}$; the increase in the drag in the same range is attributed to an increase in the strength of the vortex pair $[8]$. Angles between $16^{\circ}$ and $22^{\circ}$ show a progressive decrease in the downforce, suggesting separation of the flow at the diffuser inlet as the local pressure gradient becomes more severe. The flow visualization reported in reference $[\mathbf{8}$ ] identified a separation bubble present in similar circumstances. The progressive reduction in the downforce arises as the separation bubble grows and the reattachment length increases. The drag variation supports this, as increased levels of drag are observed in this angle range with a maximum at $22^{\circ}$. The increased drag arises from increasing strength of the vortex structures as well as increasing separation. At $25^{\circ}$ both the downforce and the drag are reduced as the centreline separation no longer reattaches. At $28^{\circ}$ and $30^{\circ}$ the diffuser is completely stalled with much reduced drag and downforce, the two configurations producing very similar results. Where comparisons are possible, the results for the plane diffuser were compared with the results of Cooper et al. [3] in Fig. 5. A more complete comparison has been given in reference [12]. The diffuser characteristics compare well with those obtained by Cooper et al., with

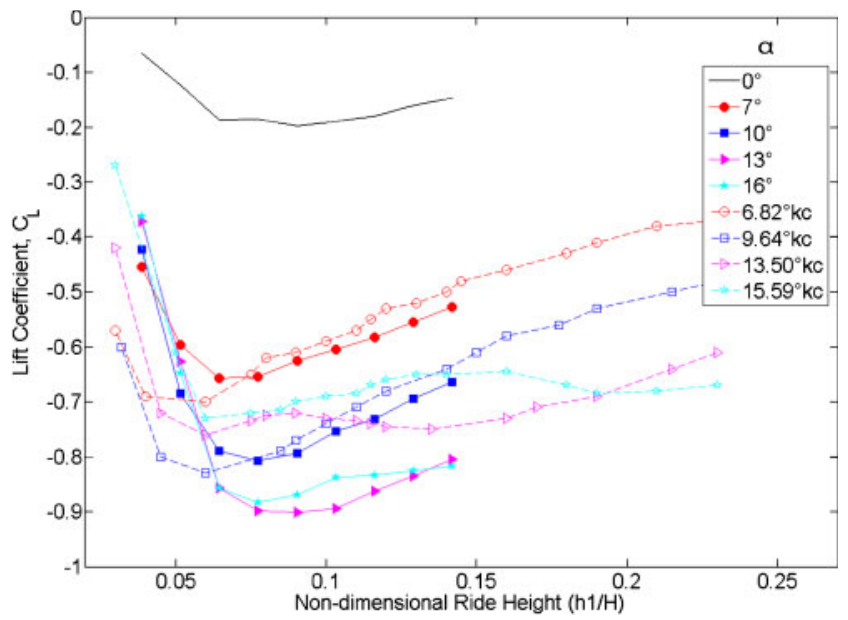

Fig. 5 Comparison with the work of Cooper et al. (indicated by kc in the key)

almost identical trends and very similar curve gradients. The primary difference is that the optimum downforce occurs at higher non-dimensional ride heights than those reported by Cooper et al. because the model, tunnel, and blockage ratios are different and the onset boundary layers are unlikely to be the same. The four ride height regions observed by Zhang and co-workers were not repeated identically here as all angles exhibited 'maximum downforce', 'downforce reduction', and 'low downforce' with the addition of 'downforce enhancement' observed at small angles. However, the non-dimensional ride heights over which these regions occurred were markedly lower than those found by Zhang and co-workers. This can be attributed to the longer length-to-width ratio of the model and diffuser length. As a large proportion of the down- 
force comes from the flat underbody, the influence of the length of this flat area and the diffuser length changes the pressure recovery distribution and therefore the variation in the downforce.

The plane diffuser performance is summarized in the contour plots in Fig. 6, presented using the nondimensional length $N / h_{1}$, which for this set of data is essentially the ride height $h_{1}$ as the diffuser length $N$ is fixed. The data are plotted in this way to be consistent with other diffuser studies. The rear-lift coefficient $C_{\mathrm{LR}}$, as opposed to the overall lift coefficient, is used, as in reference [3]. This approach highlights more specifically the diffuser contribution. Figure 6(a) shows that at all non-dimensional lengths, as the AR increases, the downforce passes through an optimum. At different diffuser angles the behaviour can be divided into three regions. The maximum downforce occurs between $13^{\circ}$ and $16^{\circ}$, and in the optimum region between $10^{\circ}$ and $22^{\circ}$ the downforce is particularly sensitive to the nondimensional length, with levels of the downforce increasing as the non-dimensional length is reduced. Within the two regions above and below this, the diffuser performance is almost independent of the non-dimensional length.

The lift-to-drag ratio (Fig. 6(b)) produces a broadly similar plot to the diffuser-based downforce, but here the optimum performance region is at $13^{\circ}$ compared with the maximum downforce at a slightly larger diffuser angle. This suggests that the additional downforce above $13^{\circ}$ arises at the expense of an increased induced drag component.

Centre-line pressure distributions for three diffuser angles are presented in Fig. 7. The angles chosen are associated with their performance in the plane

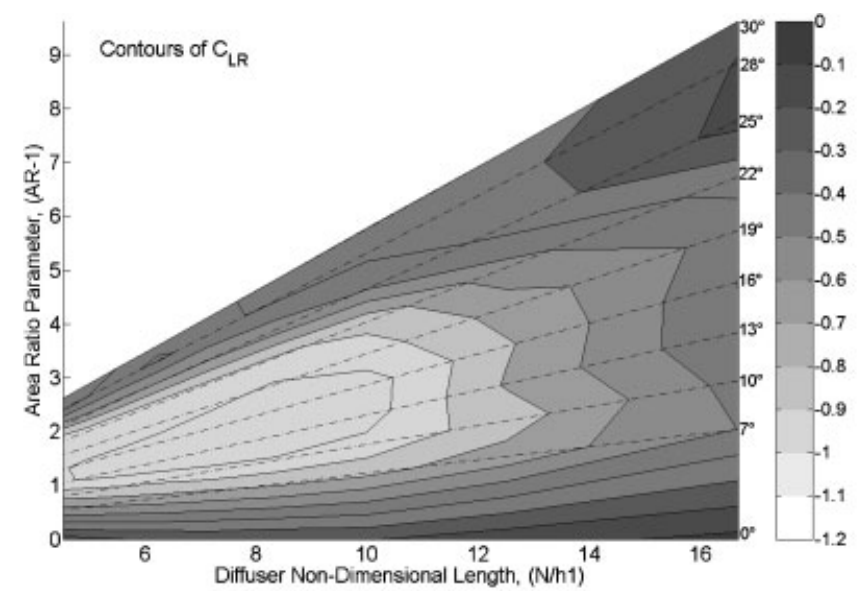

(a) diffuser configuration. An angle of $13^{\circ}$ produces the greatest downforce; an angle of $16^{\circ}$ showed similar levels of downforce but higher levels of drag than the angle of $13^{\circ}$, suggesting that it had started to separate; and an angle of $25^{\circ}$ had low levels of downforce and high levels of drag, suggesting that it was largely separated.

All three diffuser angles show a characteristic distribution around the front face of the model with a front stagnation that moves towards the ground as the ride height is reduced, as shown in Fig. 8. The underbody flow accelerates around the lower radius of the front face, producing a significant depression. Forward from $x / L=0.4$ the flat underbody pressure becomes increasingly negative as the ride height is reduced and the pressure recovery is suppressed. Downstream of $x / L=0.4$ the underbody pressure is dependent on the diffuser pumping. Decreasing the ride height increases the $\mathrm{AR}$, increasing the diffuser pressure recovery. This is seen as the characteristic depression at the diffuser inlet $(x / L=0.75)$. The distributions in Fig. 7 are consistent with those observed by Cooper et al. [3]. Additionally, around $x / L=0.1$ there is evidence of a small separation bubble just after the lower front-edge radius. This was identified in reference [4] in a more pronounced way and was attributed to the strong adverse pressure gradient around the front-edge radius.

Within the $13^{\circ}$ diffuser (Fig. 7(a)) the pressure recovery is close to ideal, suggesting that it is largely attached. As the ride height is reduced, the depression at the inlet is increased (a more negative pressure coefficient), giving rise to an increased downforce similar to that seen in reference [9]. At $16^{\circ}$ (Fig. 7(b)) the pressure indicates a probable separa-

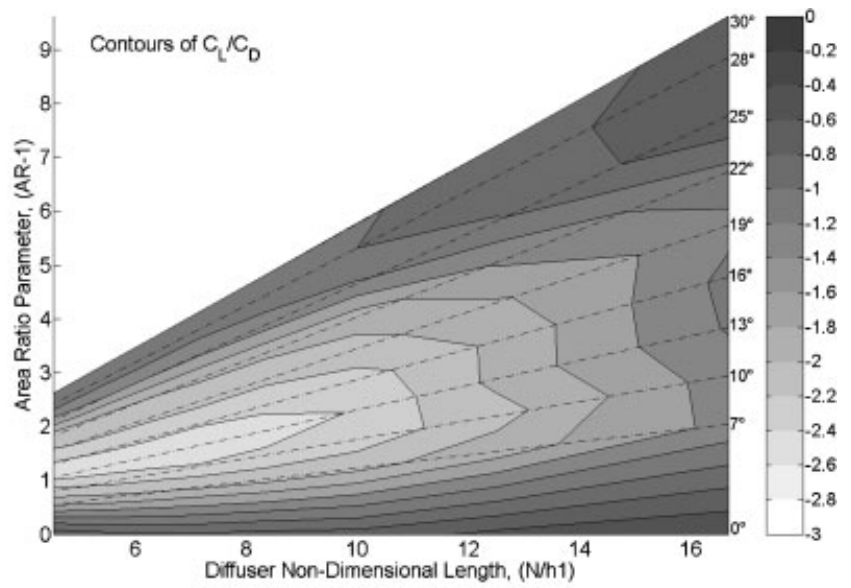

(b)

Fig. 6 Contours of (a) the diffuser-based downforce and (b) the lift-to-drag ratio for the plane diffuser 


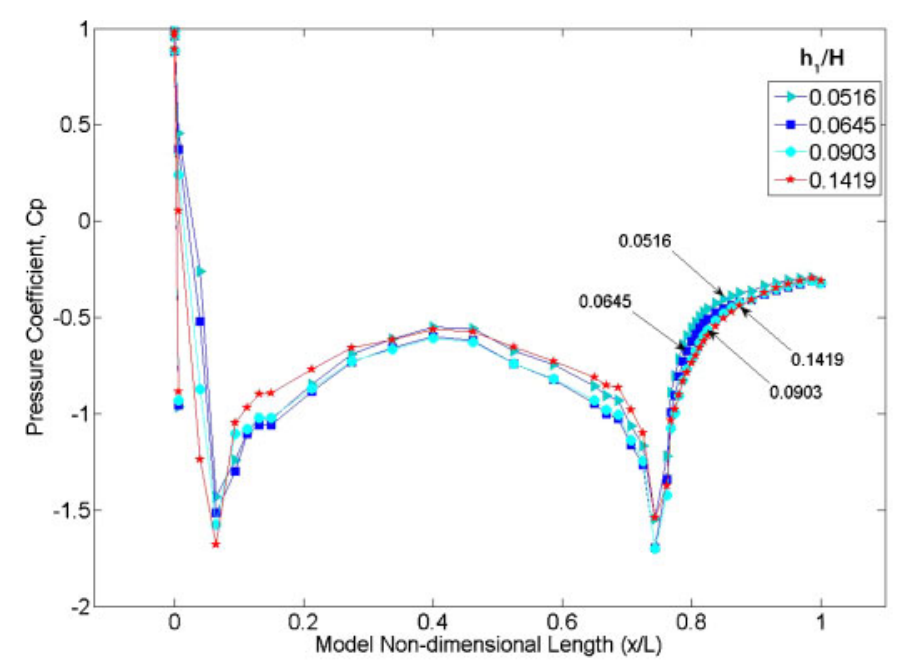

(a)

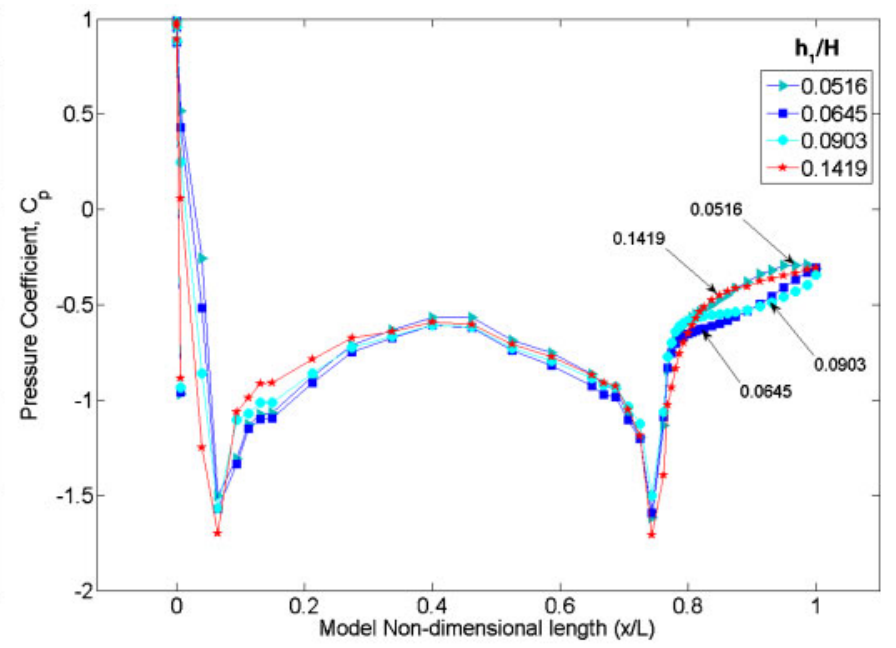

(b)

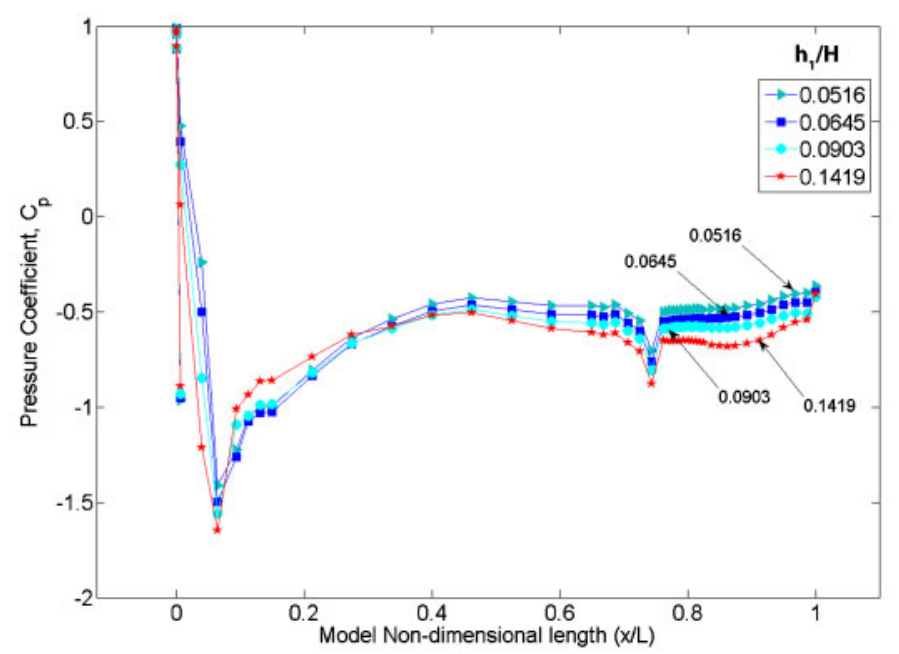

(c)

Fig. 7 Pressure distributions for (a) the $13^{\circ}$, (b) the $16^{\circ}$, and (c) the $25^{\circ}$ plane diffusers

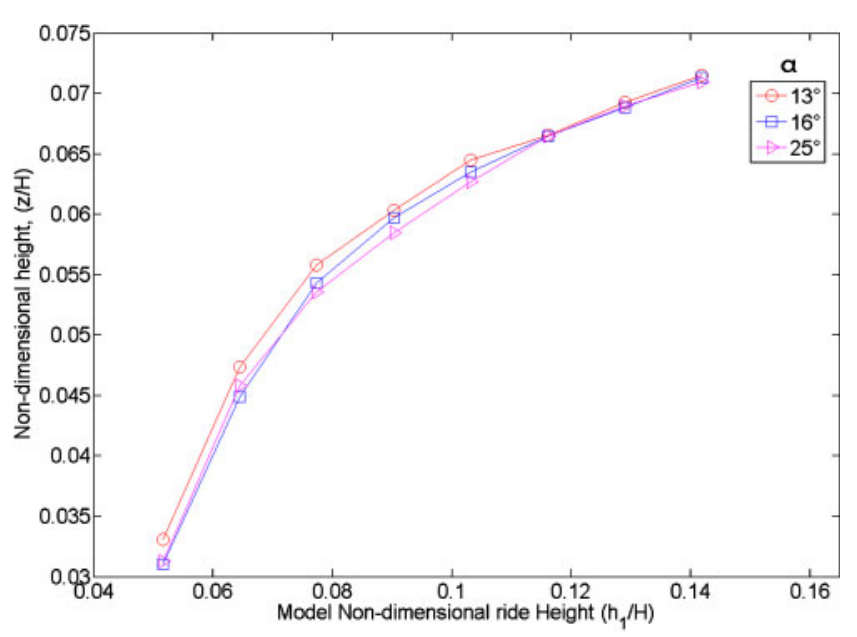

Fig. 8 Stagnation tion close to the diffuser inlet at $x / L=0.79$, shown by the plateau region [13]. The force measurements support this (Fig. 4) as the increased drag in the $16^{\circ}$ diffuser, compared with the $13^{\circ}$ diffuser, is assumed to be attributed to separation rather than to the drag associated with the increase in the vortex strength, because the downforces of the two configurations have similar magnitudes. Further downstream of the inlet, pressure recovery continues to the base pressure. As the ride height is increased, the adverse pressure gradient is reduced and the flow is less susceptible to separation at the inlet. At $25^{\circ}$ (Fig. 7 (c)) the separation point has moved upstream and occurs at the inlet, with the diffuser largely separated at all ride heights, reducing its ability to recover pressure effectively. This is confirmed by the large reduction in the downforce and the increase in 
the drag seen in Fig. 4. A small depression at the inlet occurs as the flow is locally accelerated, and the downforce gains over the flat-floor configuration are due to the upsweep and ground interaction mechanisms rather than to diffuser pumping.

\section{MULTIPLE-CHANNEL DIFFUSER PERFORMANCE}

It has been observed in conical diffusers [14] that vanes and splitters can provide improved performance through the constraint of separation and increased three-dimensional flow. While the singlechannel plane diffuser is effective, this conical diffuser behaviour suggests that the use of multiple channels could provide increased performance and usability.

The variation in the lift coefficient with the nondimensional ride height is plotted in Fig. 9. For

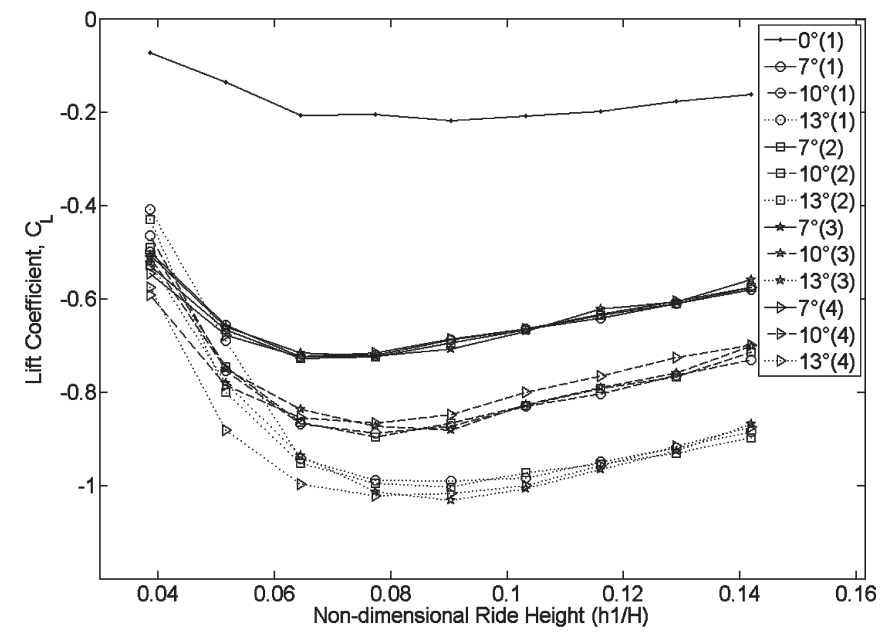

(a) clarity the results are plotted in three groups: small angles $\left(0-13^{\circ}\right)$, midrange angles $\left(16-19^{\circ}\right)$, and large angles $\left(22-30^{\circ}\right)$ for the plane diffuser configuration (1), the two-channel diffuser configuration (2), the three-channel diffuser configuration (3), and the four-channel diffuser configuration (4).

At small diffuser angles (Fig. 9(a)), all four diffuser configurations exhibit similar trends; as the number of channels is increased, the downforce is reduced marginally. As these small angles are assumed to be largely attached in the plane configuration, the division into a number of channels has no advantage in promoting improved diffuser flow. The small reductions arise because the channel splitters reduce the active area of the diffuser, and in fact the multiple-channel diffusers produce greater downforce per unit area. The exceptions to this trend are the $13^{\circ}$ diffusers, the multiple-channel configurations producing a slightly greater downforce, parti-

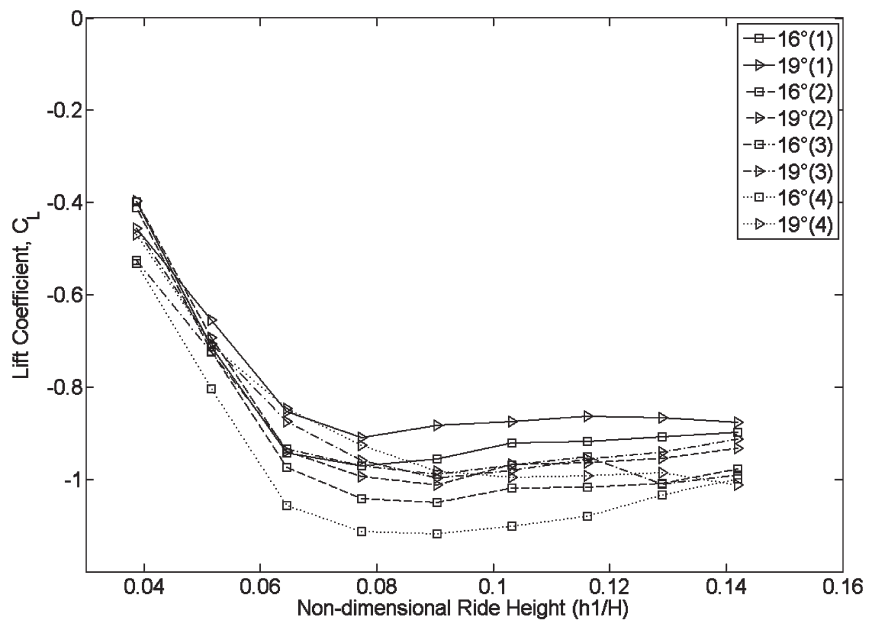

(b)

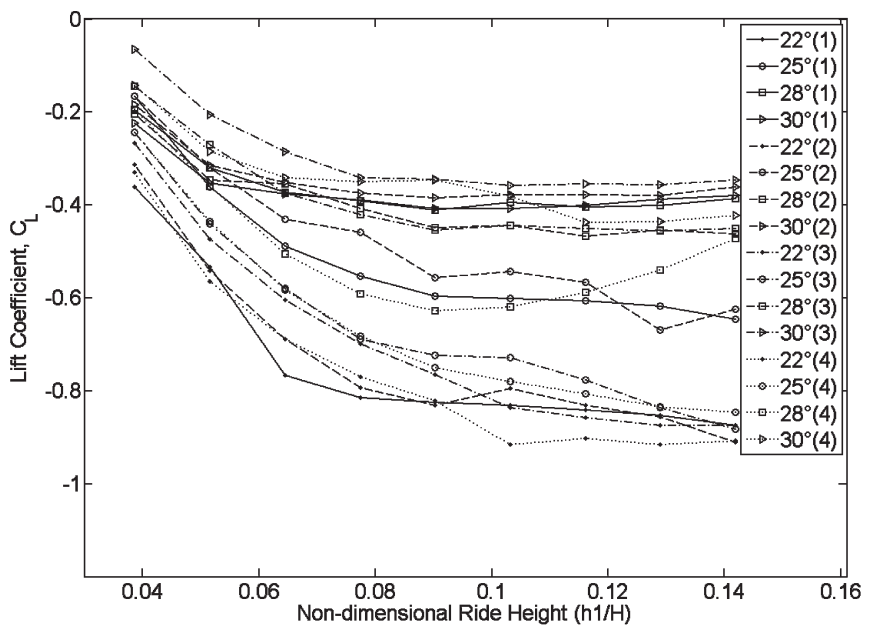

(c)

Fig. 9 The lift coefficient plotted against the non-dimensional ride height for (a) small, (b) midrange, and (c) large diffuser angles 
cularly at lower ride heights. As the plane diffuser is largely attached, the improvement must be due to an improvement in one of the downforce mechanisms, rather than to changes in the separation characteristics, due to the drag reduction observed, rather than increasing, which would be seen if the vortex strength increased. At midrange angles (Fig. 9(b)), the differences between the plane and multiplechannel diffusers is more pronounced. While the overall trends are the same, splitting the diffuser gives an improvement in the downforce due to an increase in the diffuser pumping contribution. As separation was present in the plane diffuser (Fig. 7), increased diffuser pumping results in an increase in the vortex strength, similar to that seen in reference [8] where a change in the diffuser pumping due to a change in the ride height resulted in an increase in the vortex strength. This increased vortex strength can aid flow attachment and improve the downforce [5]. In this midrange the total downforce is increased by 13 per cent compared with the optimum plane diffuser, significantly extending the performance envelope. At the largest angles (Fig. 9(c)), the difference between the configurations is much larger, with the multiple-channel diffusers working much more efficiently than the plane diffuser does. This improvement is attributed to reduced levels of separation

The contours of the diffuser-based downforce for multiple-channel diffusers were found to show similar trends to those seen for the plane diffuser (Fig. 6) and are therefore not included. The change in the lift coefficient between the plane and multiple-channel diffusers is defined by

$$
\Delta C_{\mathrm{L}}=C_{\mathrm{L}(\text { multiple-channel })}(\alpha)-C_{\mathrm{L}(\text { plane })}(\alpha)
$$

and the contours are shown in Fig. 10. For all multiple-channel diffusers, much of the figure shows little or no performance improvement, with the

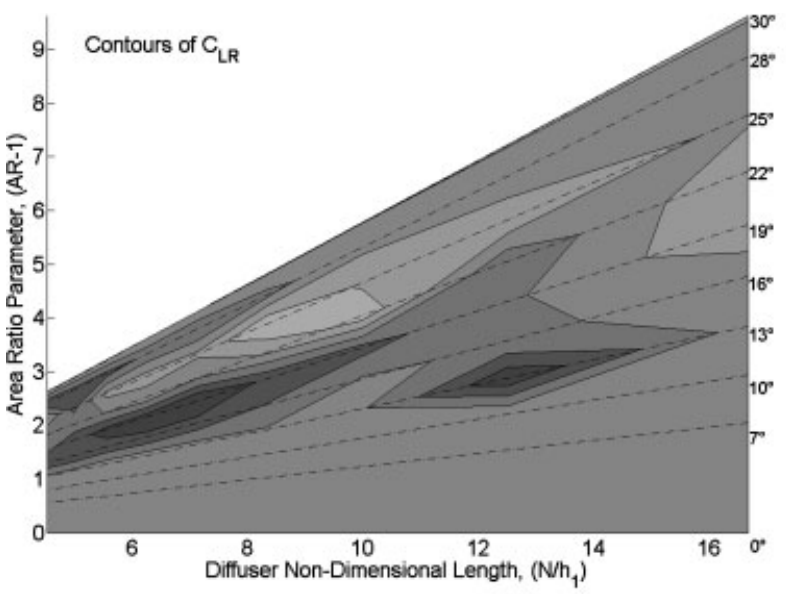

(a)

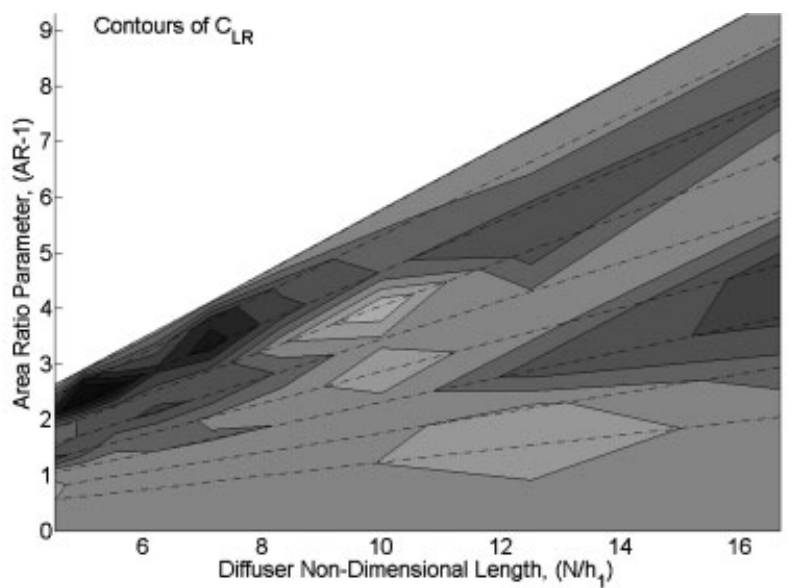

(b)

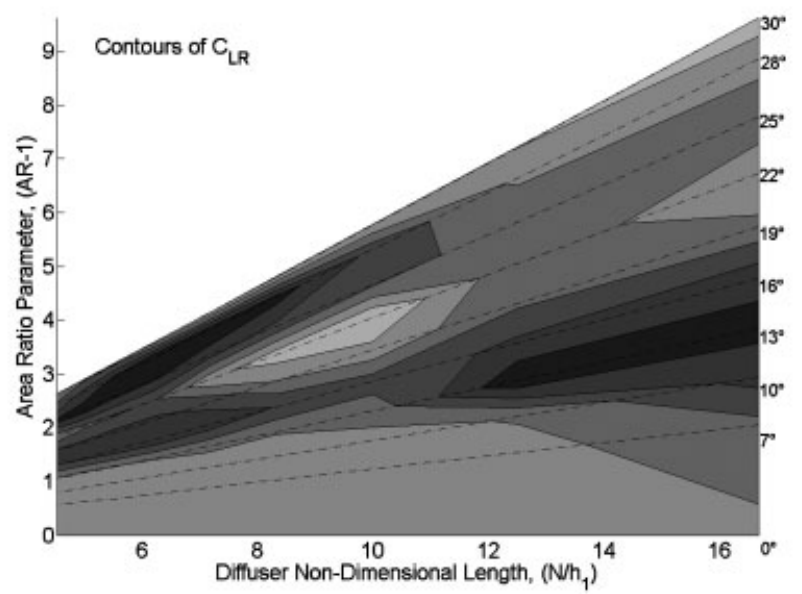

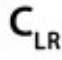

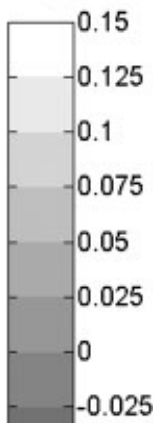

025 0.05 0.075

(c)

Fig. 10 Contours of the difference in the diffuser-based downforces for (a) the two-channel, (b) the three-channel, and (c) the four-channel diffusers 
changes sufficiently small $( \pm 0.05)$ not to have a significant effect on road vehicle handling, although the gains might be considered important in the ongoing development of a race car. However, in some parts of the figure the changes are much greater than this. In the two-channel diffuser (see Fig. 10(a)) there are much larger gains, up to $C_{\mathrm{L}}=0.125$ at midrange to large angles for a small range of ARs and ride heights. For the three-channel diffuser (Fig. 10(b)), improvements are observed at high ARs, predominately at an angle of around $25^{\circ}$, where the improvement is between 0.150 and 0.20 . The four-channel diffuser (Fig. 10(c)) produces the greatest performance improvement covering two distinct regions: one of high ARs and high ride heights, and the other of medium ARs and low ride heights. In these areas the downforce is increased by up to 0.200 . An improvement of $0.15-0.20$ represents a 5-7 per cent improvement for a Formula 1 car with an overall lift coefficient $C_{\mathrm{L}}=-3$, and should be viewed in the context of typically $6-10$ per cent [2] improvement over a full race season.

Similar to the diffuser-based downforce, the change in the lift-to-drag ratio was calculated, and the contours are shown in Fig. 11. It was identified that, for the two- and three-channel diffusers, the improvement is limited to distinct regions; the twochannel (Fig. 11(a)) improvement is seen at $16-19^{\circ}$ at high ride heights, while the three-channel (see Fig. 11(b)) improvement occurs between $22^{\circ}$ and $28^{\circ}$ and between $10^{\circ}$ and $16^{\circ}$ at low ride heights. The four-channel diffuser (Fig. 11(c)) shows large improvements in the majority of configurations. The gains occur mainly at large angles and high ride heights although improvement is seen at the midrange angles which have not previously been identified in the other configurations. These regions of performance improvement occur where the plane diffuser begins to be compromised by the onset of separation, as identified in Fig. 9(b).

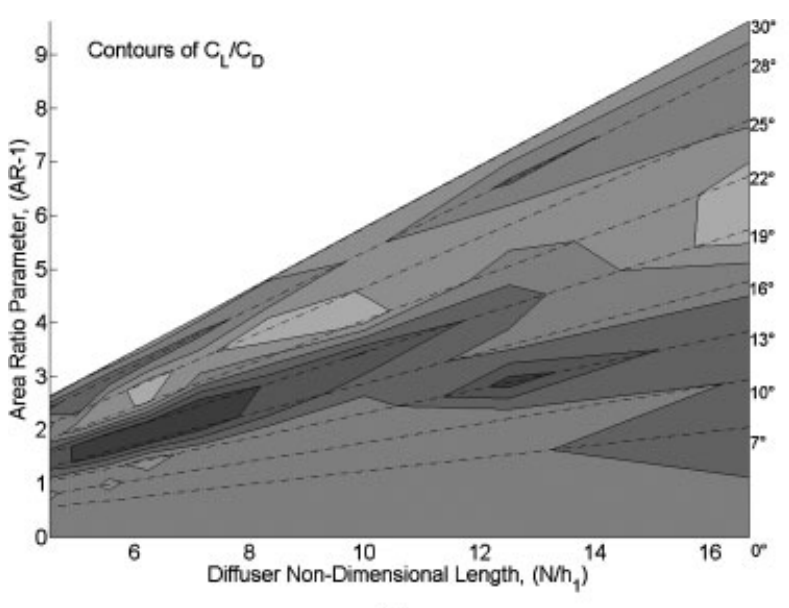

(a)

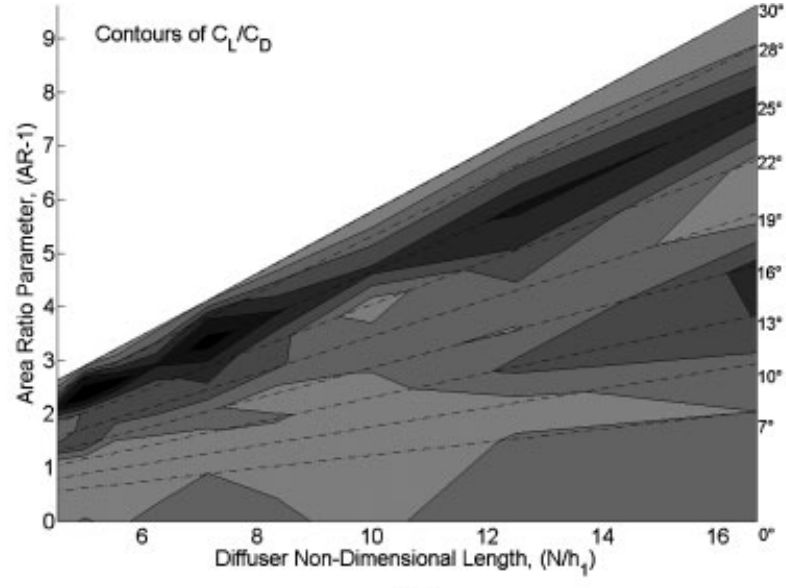

(b)
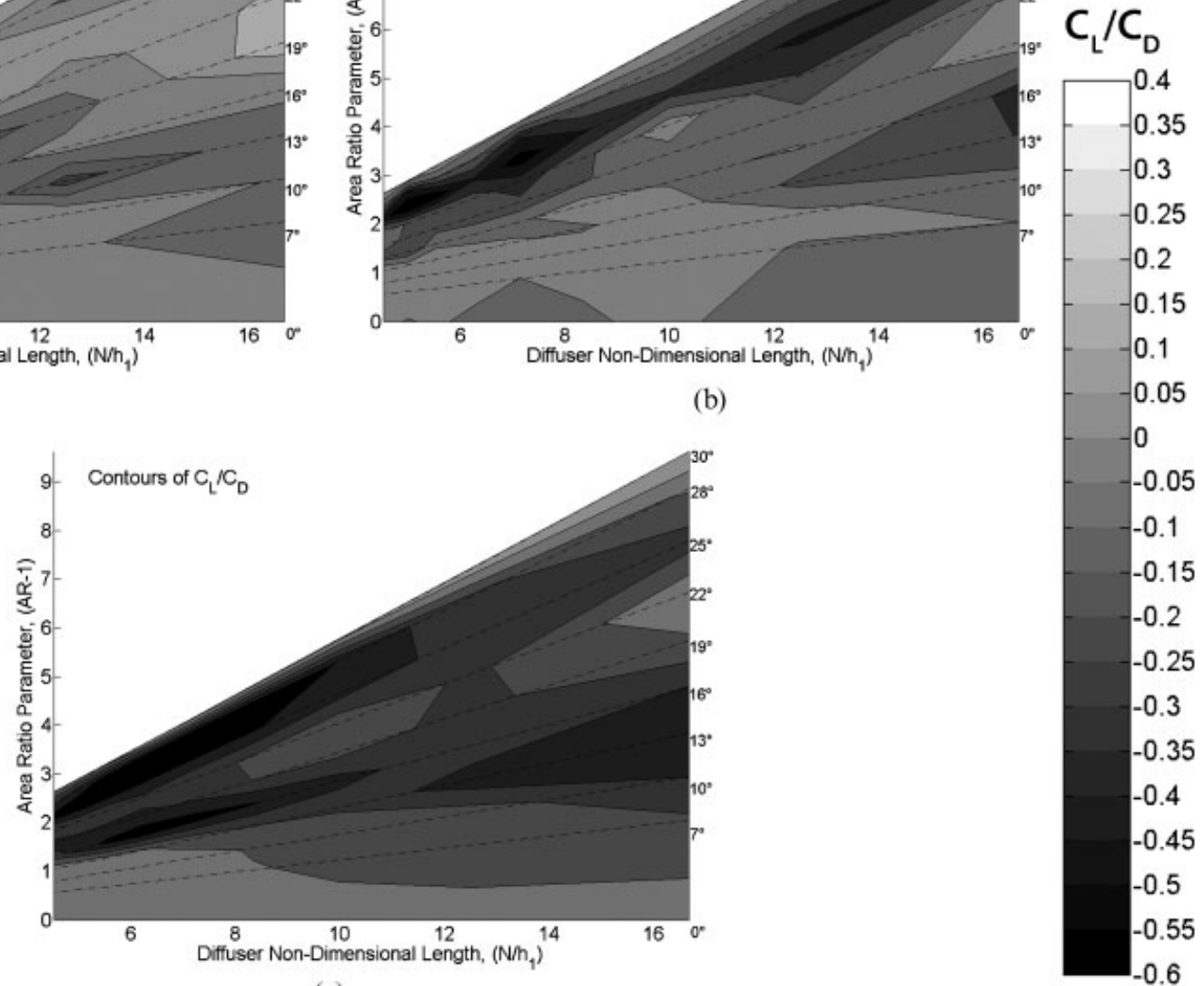

(c)

Fig. 11 Contours of the difference in the lift-to-drag ratios for (a) the two-channel, (b) the threechannel, and (c) the four-channel diffusers 
Centre-line pressure data are less useful for the multiple-channel configurations because the centreline of the model is the location of a splitter plate in some cases, and the centre-line of each channel is in a different lateral location. Pressure data for the multiple-channel configurations (and plane diffuser) are therefore presented in the following figures in the form of contour plots. The plots begin just upstream of the diffuser inlet $(x / L=0.75)$ and show an area of half the model width which therefore covers a different number of channels in each configuration: half in the plane diffuser, one in the two-channel diffuser, one and a half in the threechannel diffuser, and two in the four-channel diffuser. The end plates $(y / L=0.25)$ and splitter plates $(y / L=0, y / L=0.114$, and $y / L=0.152)$ are shown in the figure for clarity. Three diffuser angles are presented, namely $13^{\circ}, 16^{\circ}$, and $25^{\circ}$, at a single ride height $h_{1} / H=0.0903(28 \mathrm{~mm})$. The area map pressure tapping positions are shown in the figures as open circles.

The $13^{\circ}$ data (Fig. 12) all show areas of low pressure at the inlet $(x / L=0.75)$ corresponding to the diffuser pumping depression observed in the centreline pressure distributions (Fig. 7). Low pressures are also seen near the end plates $(y / L=0.25)$ at the location of the vortex structure. At the exit of the diffuser the pressure is close to the base pressure.

The plane diffuser (Fig. 12(a)) and two-channel diffuser (Fig. 12(b)) show very similar distributions,

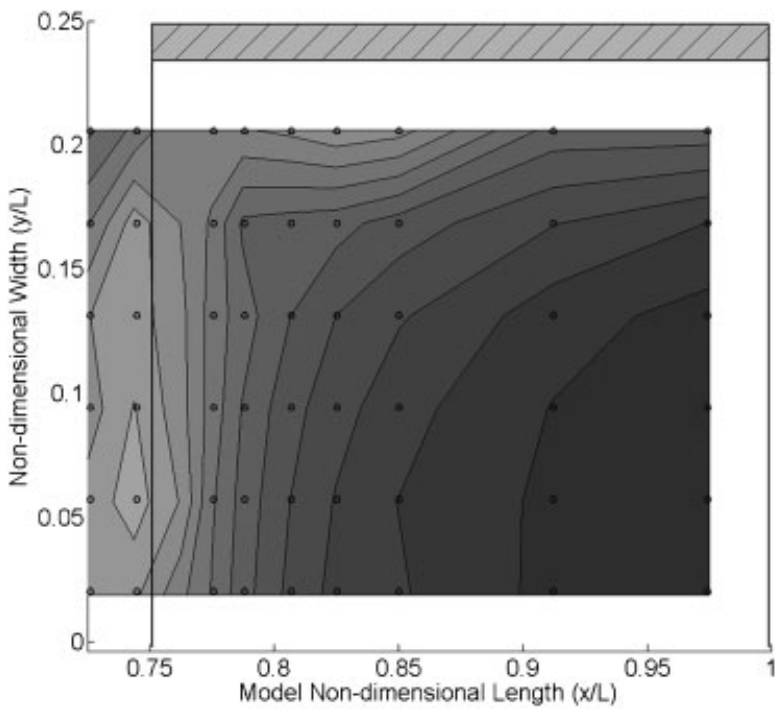

(a)

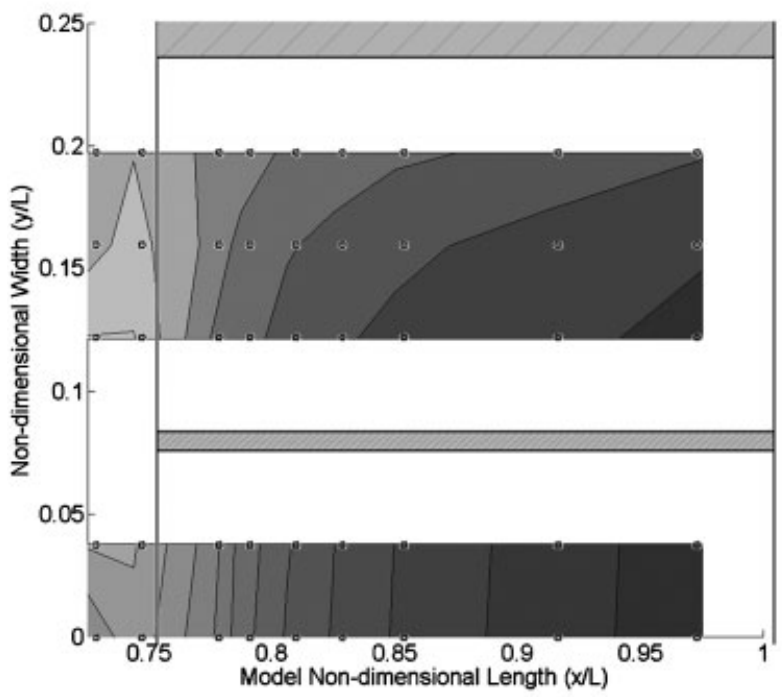

(c)

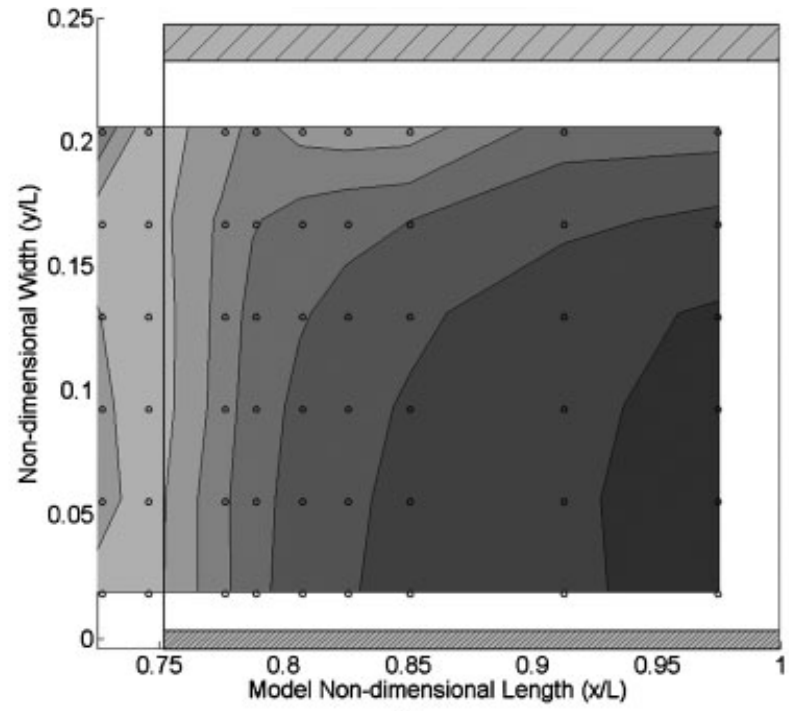

(b)

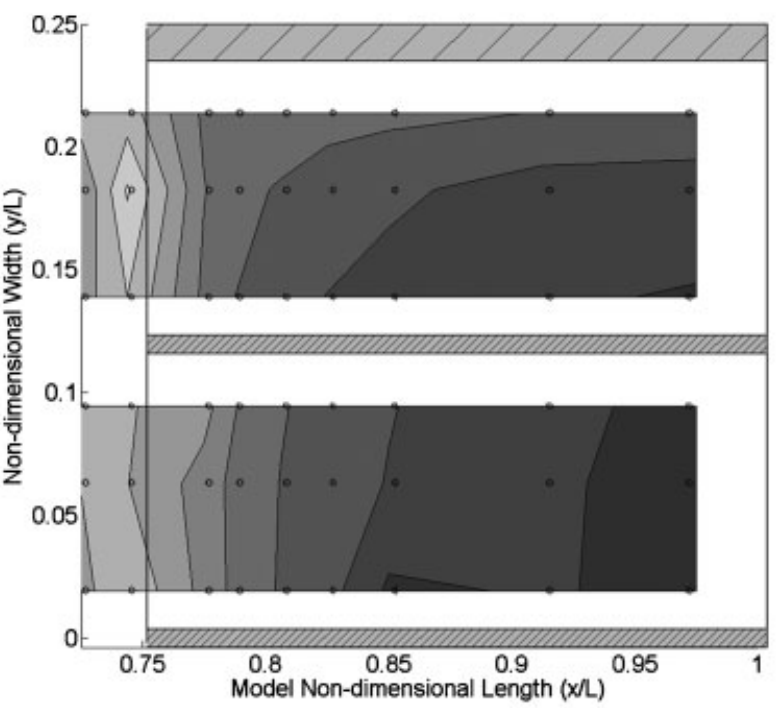

(d)

Fig. 12 Contours of the pressure for (a) the plane, (b) the two-channel, (c) the three-channel, and (d) the four-channel $13^{\circ}$ diffusers at $28 \mathrm{~mm}(x / L=0.0903)$ 
the primary difference being that lower pressures at the inlet are observed in the two-channel diffuser from an increased diffuser pumping component. This results in greater inflow into the diffuser channel 'feeding' the vortex structure, shown by lower end-plate region pressures. The presence of the splitter plate gives lower pressures at the inlet and end-plate regions. In reference [9], surface flow visualization showed S-shaped lines to be more pronounced as the vortices increased in strength, with areas of lower pressure observed around the end plates similar to that seen here. Therefore, it is assumed that the splitter plates help to increase the vortex strength by constraining it into a smaller diffuser channel, similar to the containment of separation seen in conical diffusers with vanes and splitters [14]. The strengthening of the vortex structures makes them more resistant to breakdown and aids attachment, resulting in a higher downforce. As the non-dimensional length is increased, the number of contours passed through is reduced, showing a more gradual pressure recovery and greater downforce, confirmed by the force measurements (Fig. 9) which gave a 1.4 per cent improvement for this configuration.

The three-channel configuration (Fig. 12(c)) and four-channel configuration (Fig. 12(d)) each show an inside diffuser channel and an outside diffuser channel. In both cases the outside channels perform better with greater diffuser pumping at the inlet, with the three-channel exhibiting the greatest diffuser pumping of all configurations. This increase in diffuser pumping results in stronger vortex structures which are then constrained owing to the decreased channel width. The combination of increased diffuser pumping and upwash results in the increases in the downforce observed in the force measurements of 4.4 per cent and 2.7 per cent for the three-channel diffuser and four-channel diffuser respectively. The inside channels for the threeand four-channel diffusers have similar distributions to the equivalent position on the plane and twochannel configurations respectively, despite the presence of the splitter plates. This suggests that these distributions are independent of the presence of vortex structures and therefore are affected primarily by the upsweep component and pressure gradient such that, if separation occurs, it is likely to occur in this region.

Increasing the diffuser angle to $16^{\circ}$ (Fig. 13) shows only relatively small differences compared with the $13^{\circ}$ diffusers; similar variations are observed but with less diffuser pumping at the inlet. The two-channel diffuser has greater diffuser pumping than the plane diffuser, leading to increased vortex strength, which is identified by the lower pressures in the end-plate area. The centre-line pressure measurements (Fig. 7) identified separation in the plane configuration; however, a more gradual pressure recovery is observed for the two-channel diffuser and hence less separation has occurred because the increase in the vortex strength gives a 10 per cent improvement in the downforce.

The three-channel diffuser (Fig. 13(c)) and fourchannel diffuser (Fig. 13(d)) perform better than the plane diffuser, with much greater diffuser pumping at the inlet in both the inside channels and the outside channels. The lower pressures and more gradual pressure recovery than for the plane and two-channel diffusers suggest reduced separation or adverse pressure gradient. The increased diffuser pumping in the outside channel has the same effect as was observed at $13^{\circ}$ but additionally the vortices help to reduce or even eliminate the separation observed in the plane diffuser. The improvement in performance is confirmed by the force measurements where an increase of up to 17 per cent in the downforce is observed. This change around the critical angle is important because it demonstrates the potential to extend the performance envelope close to the plane diffuser optimum.

As the diffuser angle is increased further to $25^{\circ}$, a transition in behaviour between configurations is observed. The plane diffuser (Fig. 14(a)) and twochannel diffuser (Fig. 14(b)) no longer exhibit the strong pressure recovery seen at smaller angles, suggesting that vortex breakdown may have occurred, causing a small amount of asymmetry in the flow as observed in the surface flow visualization in reference [9]. This is consistent with the large reductions in the downforce and drag observed in Fig. 4.

As the number of channels is increased, the asymmetry disappears and the three- and fourchannel diffusers perform much better. The inside channels appear to remain completely stalled. The improvement in the downforce comes from the outside channels, which show similar characteristics to those for smaller angles, with strong diffuser pumping and gradual pressure recovery to the base pressure. Unlike the plane diffuser at $28^{\circ}$, the threeand four-channel configurations show the presence of vortex structures, albeit weaker than those at smaller angles. The presence of splitter plates makes the configurations less susceptible to flow separation owing to increased diffuser pumping and vortex 


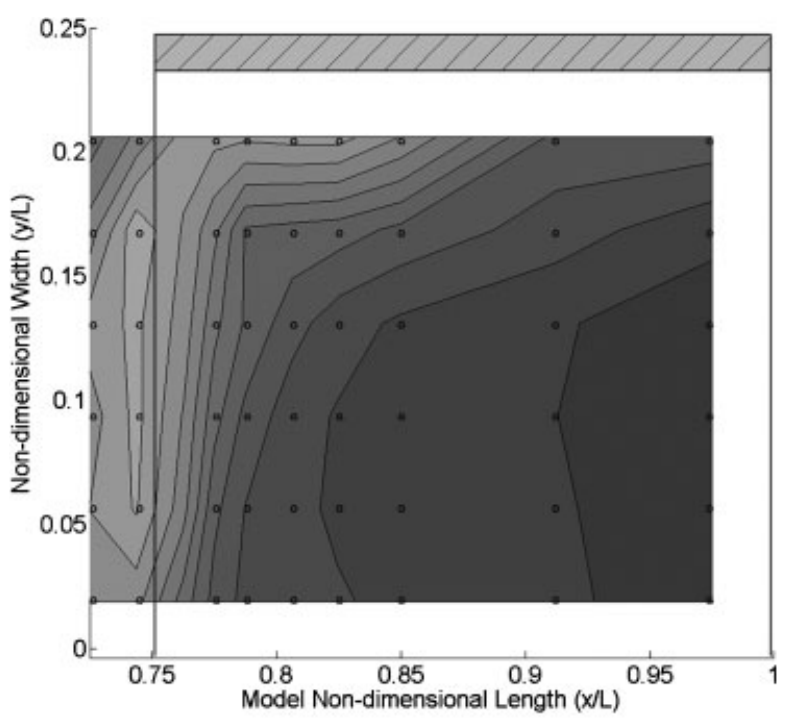

(a)

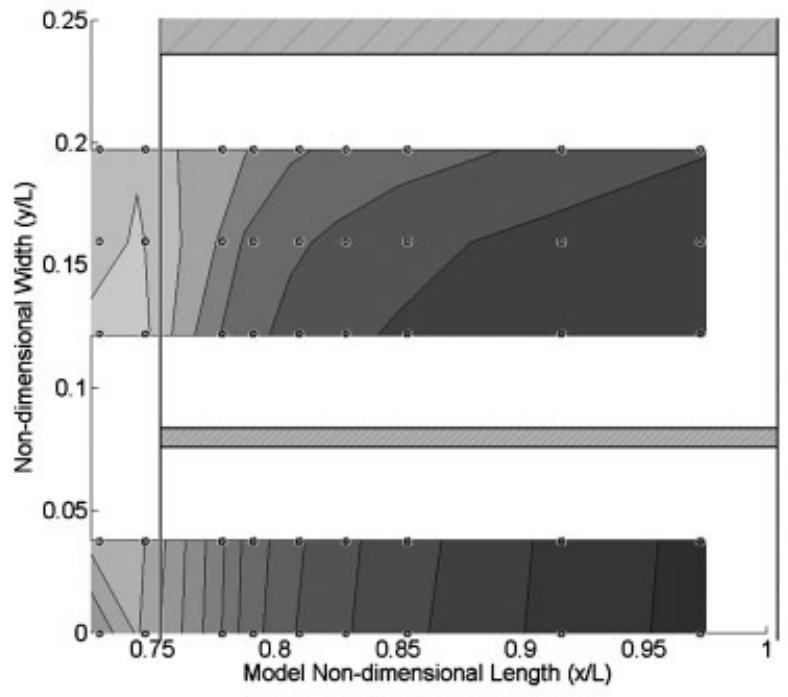

(c)

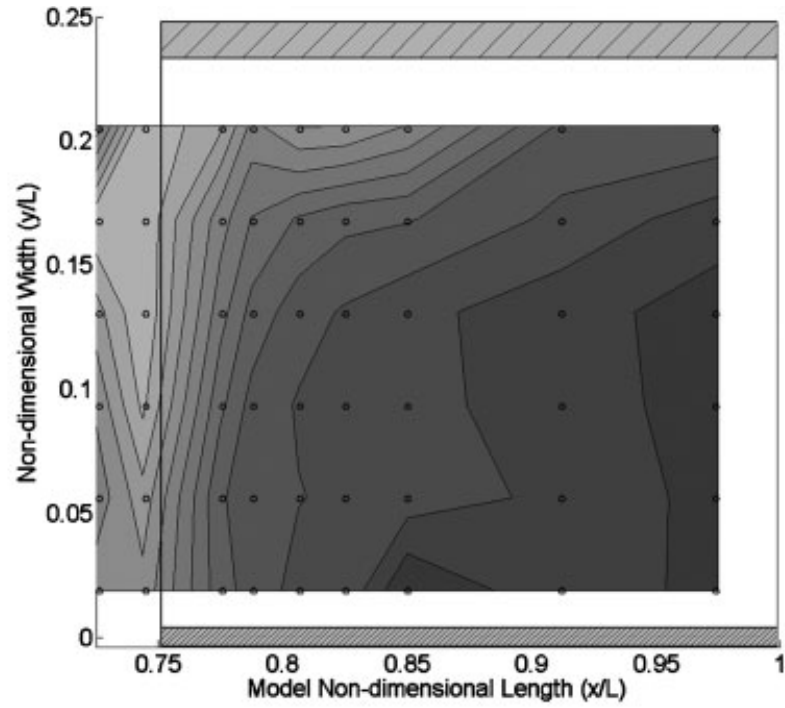

(b)

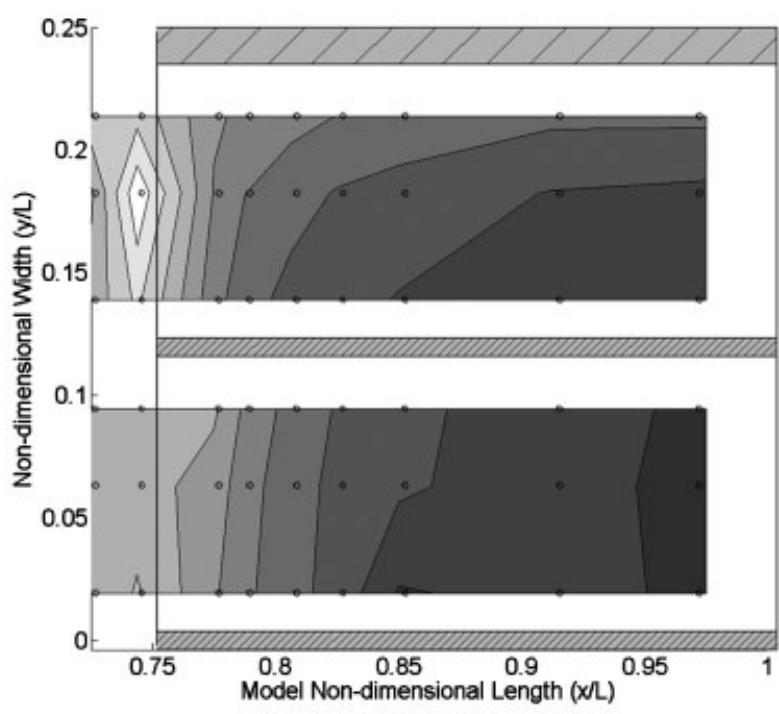

(d)

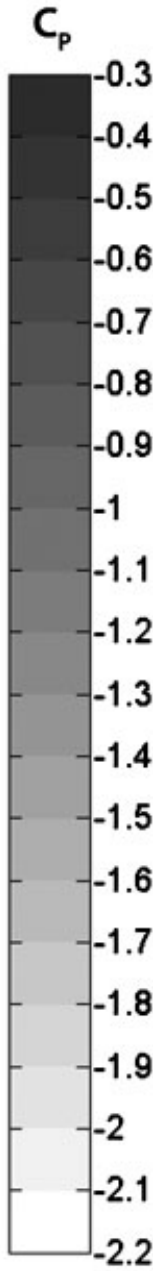

$-2.2$

Fig. 13 Contours of the pressure for (a) the plane, (b) the two-channel, (c) the three-channel, and $(\mathrm{d})$ the four-channel $16^{\circ}$ diffusers at $28 \mathrm{~mm}(x / L=0.0903)$

strength and hence improved downforce. This is confirmed by the force measurements, which give 21 per cent and 26 per cent increases in the downforce. It must be noted that, although a performance improvement is observed with the three- and fourchannel configurations, the levels of downforce produced remain relatively low.

\section{CONCLUSIONS}

1. An investigation into the performance of plane and multiple-channel diffusers has been carried out using force and pressure measurements. Ten diffuser angles and eight ride heights were investigated.

2. The plane diffuser showed similar trends in the lift and drag to published data. The optimum angle for the downforce lies between $13^{\circ}$ and $16^{\circ}$. Above $16^{\circ}$ the downforce levels are reduced with a corresponding increase in the drag.

3. The centre-line pressure distributions indicate that, above $13^{\circ}$, local separation occurs at the diffuser inlet and that, at $25^{\circ}$ and above, the diffuser is stalled although it continues to generate a downforce through the mechanism of upsweep.

4. The multiple-channel diffusers showed similar trends in the lift and drag to the plane diffuser 


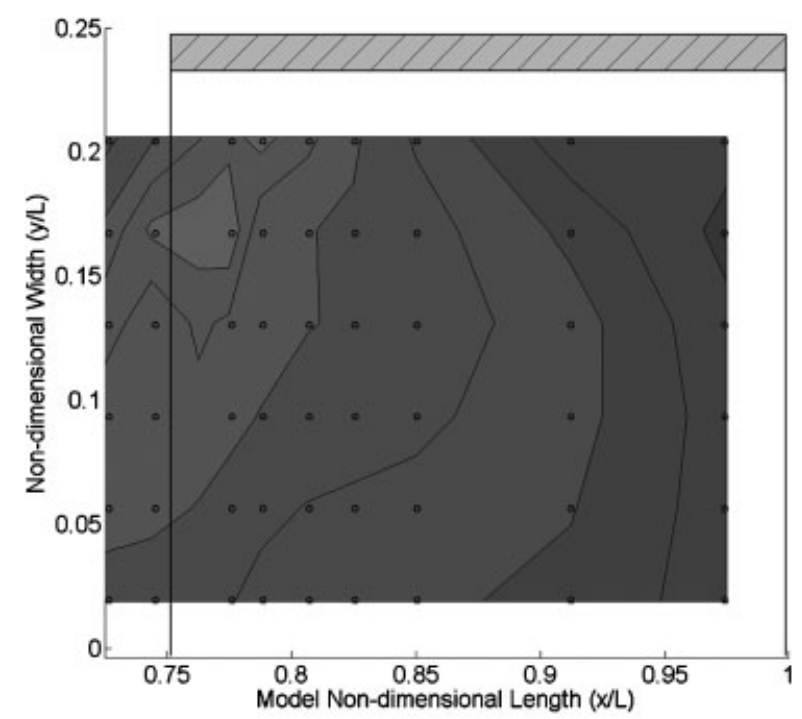

(a)

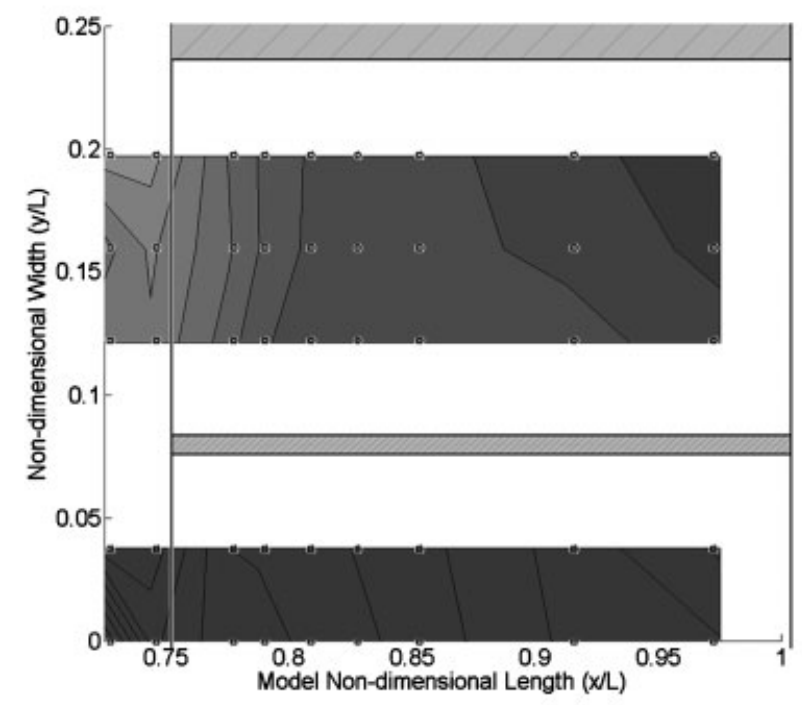

(c)

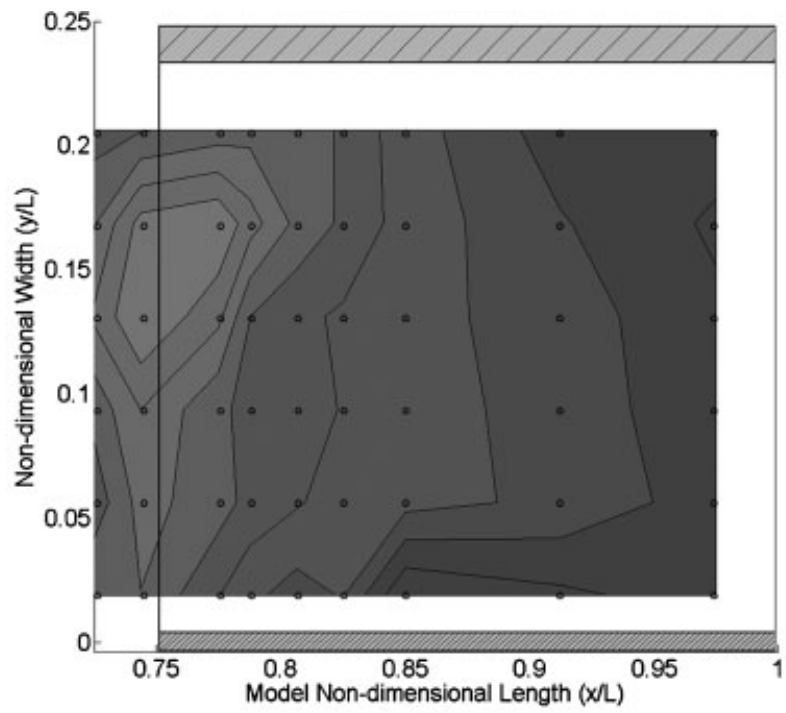

(b)

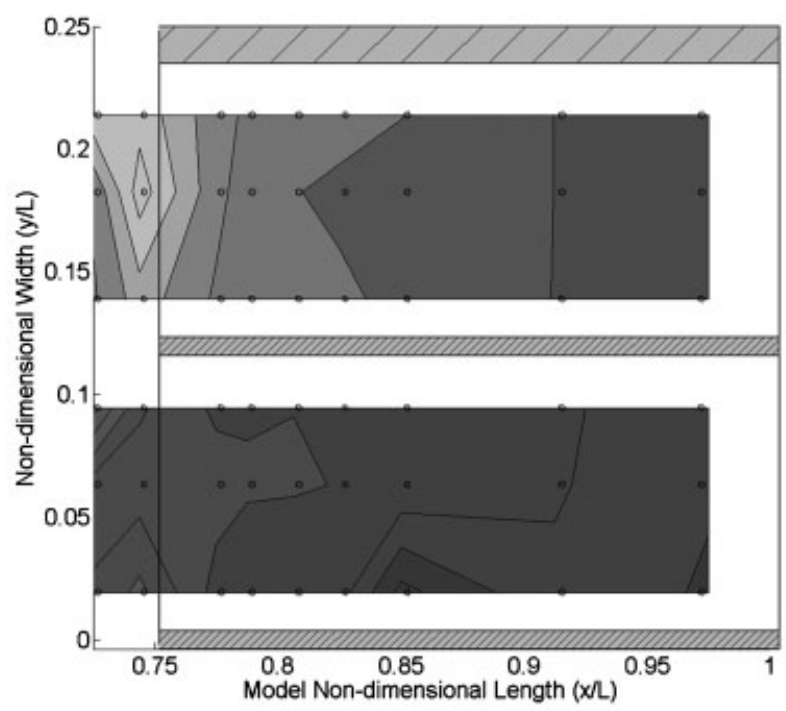

(d)

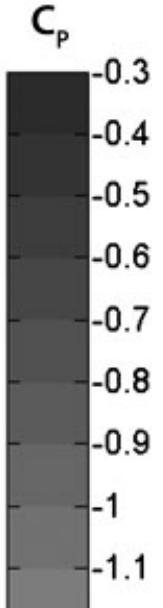

$-1.2$

$-1.3$

1.4

$-1.5$

$-1.6$

$-1.7$

$-1.8$

$-1.9$

$-2$

$-2.1$

Fig. 14 Contours of the pressure for (a) the plane, (b) the two-channel, (c) the three-channel, and (d) the four-channel $25^{\circ}$ diffusers at $28 \mathrm{~mm}(x / L=0.0903)$

and, for angles of $13^{\circ}$ and above, the multiplechannel configurations show an improved downforce, with the percentage gains increasing with increasing diffuser angle.

5. For the midrange angles $\left(16-19^{\circ}\right)$ where the degree of separation is small, the multiplechannel configurations show large improvements in the downforce with minimal increase in the drag. In this range the total downforce is increased by 13 per cent compared with the optimum plane diffuser, significantly extending the performance envelope. The pressure maps indicate that the gains occur through improved diffuser pumping and pressure recovery in both the inner channel and the outer channel.
6. Above $19^{\circ}$, large improvements in performance are observed compared with the plane configuration, particularly for three- and four-channel setups; however, the levels of downforce produced remain relatively low. The pressure data indicate that the gains arise from improved pressure recovery in the outer channels.

\section{ACKNOWLEDGEMENTS}

The authors would like to thank Rob Hunter, Keith Coulthard, Peter Stinchcombe, and Stacey Prentice for their excellent technical support throughout this work. 
(c) Authors 2010

\section{REFERENCES}

1 Hucho, W.-H. Aerodynamics of road vehicles, 4th edition, 1998, p. 47 (SAE International, Warrendale, Pennsylvania).

2 Wright, P. Formula 1 technology, 2001 (SAE International, Warrendale, Pennsylvania).

3 Cooper, K. R., Bertenyi, T., Dutil, G., and Syms, J., and Sovran, G. The aerodynamic performance of automotive underbody diffusers. SAE paper 980030, 1998.

4 Cooper, K. C., Syms, J., and Sovran, G. Selecting automotive diffusers to maximise underbody downforce. SAE paper 2000-01-0354, 2000.

5 Ahmed, S., Ramm, G., and Faltin, G. Some salient features of the time-averaged ground vehicle wake. SAE paper 840300, 1984.

6 Howell, J. P. The influence of ground simulation on the aerodynamics of simple car shapes with an underfloor diffuser. In Proceedings of the Road Vehicle Aerodynamics Conference, Loughborough, UK, 18-19 July 1994.

7 George, A. R. and Donis, J. Flow patterns, pressures and forces in the underside of idealised ground effect vehicles. In Proceedings of the ASME Fluids Engineering Division Symposium on Aerodynamics of transportation II, December 1983, vol. 7, pp. 69-79 (ASME International, New York).

8 Zhang, X., Senior, A., and Ruhrmann, A. Vortices behind a bluff body with an upswept aft section in ground effect. Int. J. Heat Fluid Flow, 2004, 25(1), $1-9$.

9 Senior, A. and Zhang, $\mathbf{X}$. The force and pressure of a diffuser-equipped bluff body in ground effect. Trans. ASME, J. Fluids Engng, 2001, 123(1), 105111.

10 Ruhrmann, A. and Zhang, X. Influence of diffuser angle on a bluff body in ground effect. Trans. ASME, J. Fluids Engng, 2003, 125(2), 332-338.
11 Cogotti, A. A parametric study on the ground effect of a simplified car model. SAE paper 980031, 1998.

12 Jowsey, L. and Passmore, M. A. A parametric study of plane and dual-channel diffusers. In Proceedings of the Sixth MIRA International Vehicle Aerodynamics Conference, Gaydon, Warwickshire, UK, 25-26 October 2006, pp. 270-278.

13 Tani, I. Low-speed flows involving bubble separation. Prog. Aerospace Sci., 1964, 5, 70-103.

14 ESDU, Introduction to design and performance data for diffusers. Data Sheet 76027, Engineering Sciences Data Unit, London, UK, 1976.

\section{APPENDIX}

\section{Notation}

$\mathrm{AR}$

A

$A_{1}$

$A_{2}$

$C_{\mathrm{D}}$

$C_{\mathrm{L}}$

$C_{\mathrm{LR}}$

$C_{\mathrm{P}}$

$h_{1}$

$h_{1} / H$

$h_{2}$

H

$L$

$N$

$N / h_{1}$

$x$

$x / L$

$y$

$y / L$

$\alpha$ area ratio

frontal area $\left(\mathrm{m}^{2}\right)$

inlet area $\left(\mathrm{m}^{2}\right)$

exit area $\left(\mathrm{m}^{2}\right)$

drag coefficient

lift coefficient

rear-lift coefficient

pressure coefficient

ride height $(\mathrm{m})$

non-dimensional ride height

exit height (m)

model height (m)

model length (m)

diffuser length (m)

non-dimensional diffuser length

distance along the model length (m)

non-dimensional model length

distance along the model width (m)

non-dimensional model width

diffuser angle (deg) 